\title{
On Basicity of the System of Exponents with Linear Phase in Morrey-Lebesgue Space
}

\author{
Telman Gasymov $^{1 *}$ and Aida Quliyeva ${ }^{2}$ \\ ${ }^{1}$ Institute of Mathematics and Mechanics of NAS of Azerbaijan Az1141, B.Vahabzade 9, Baku \\ Email: telmankasymov@rambler.ru \\ ${ }^{2}$ Institute of Mathematics and Mechanics of NAS of Azerbaijan Az1141, B.Vahabzade 9, Baku \\ Email: aidatagiyeva1984@gmail.com
}

\begin{abstract}
This work considers the Riemann boundary value problem with the piecewise continuous coefficient in Morrey-Hardy classes. Under some conditions on the coefficient, the Fredholmness of this problem is studied and the general solution of homogeneous and non-homogeneous problems in Morrey-Hardy classes is constructed. The obtained results are applied to the study of basis properties of the system of exponents with a piecewise linear phase in Morrey-Lebesgue space.
\end{abstract}

Keywords: Morrey-Hardy classes, Riemann problem, bases, double system of exponent.

\section{Introduction}

The concept of Morrey space was introduced by C. Morrey in 1938. Since then, various problems related to this space have been intensively studied. Playing an important role in the qualitative theory of elliptic differential equations (see, for example, [1-3]), this space also provides a large class of examples of mild solutions to the Navier-Stokes system [4]. In the context of fluid dynamics, Morrey spaces have been used to model fluid flow when vorticity is a singular measure supported on certain sets in $R^{n}[5]$. There appeared lately a large number of research works which considered fundamental problems of the theory of differential equations, potential theory, maximal and singular operator theory, approximation theory, etc in these spaces (see, for example, [6] and the references above). More details about Morrey spaces can be found in [7-11].

In view of the aforesaid, there has recently been a growing interest in the study of various problems in Morrey-type spaces. For example, some problems of harmonic analysis and approximation theory have been considered in [12-19].

It should be noted that when solving many problems of mathematical physics by the Fourier method [20-23], there often arise perturbed systems of sines and cosines of the following form

$$
\begin{aligned}
& \{\sin (n t+\alpha(t))\}_{n \in N}, \\
& \{\cos (n t+\alpha(t))\}_{n \in N},
\end{aligned}
$$

where $\alpha(t)=\frac{1}{2}(\beta t+\gamma), \beta, \gamma \in R-$ are real parameters ( $N$ is the set of all natural numbers). The justification of this method requires the study of basis properties (completeness, minimality, basicity) of above systems in Lebesgue and Sobolev function spaces. Their basis properties in Lebesgue spaces $L_{p}(-\pi, \pi)$ with $\gamma=0$, have been comprehensively studied in [24-33]. The weighted case of $L_{p}$ have been considered in works by E.I.Moiseev [34,35]. Basis properties of some perturbed systems of exponents in Sobolev spaces have been studied in [36-39]. To the number of the similar works the results of authors of [40-44] can also be attributed.

One of the methods to study basis properties of systems like (1), (2) is a method of boundary value problems of the theory of analytic functions. It dates back to one note by A.V. Bitsadze [45]. This method was successfully used by the authors of [24-33]. 


\section{Needful Information}

We will need some facts about the theory of Morrey-type spaces. Let $\Gamma$ be some rectifiable Jordan curve on the complex plane $C$. By $|M|_{\Gamma}$ we denote the linear Lebesgue measure of the set $M \subset \Gamma$.

The expression $f(x) \sim g(x), x \in M$, means

$$
\exists \delta>0: \delta \leq\left|\frac{f(x)}{g(x)}\right| \leq \delta^{-1}, \forall x \in M .
$$

A similar meaning will be attached to the expression $f(x) \sim g(x), x \rightarrow a$.

By the Morrey-Lebesgue space $L^{p, \alpha}(\Gamma), 0 \leq \alpha \leq 1, p \geq 1$, we mean a normed space of all functions $f(\cdot)$ measurable on $\Gamma$ equipped with a finite norm $\|\cdot\|_{L^{p, \alpha}(\Gamma)}$ :

$$
\|f\|_{L^{p, \alpha}(\Gamma)}=\sup _{B}\left(|B \bigcap \Gamma|_{\Gamma}^{\alpha-1} \int_{B \bigcap \Gamma}|f(\xi)|^{p}|d \xi|\right)^{1 / p}<+\infty .
$$

$L^{p, \alpha}(\Gamma)$ is a Banach space and $L^{p, 1}(\Gamma)=L_{p}(\Gamma), L^{p, 0}(\Gamma)=L_{\infty}(\Gamma)$. Weighted version of the MorreyLebesgue space $L_{\mu}^{p, \alpha}(\Gamma)$ on $\Gamma$ with a weight function $\mu(\cdot)$ and a norm $\|\cdot\|_{L_{\mu}^{p, \alpha}(\Gamma)}$ can be defined in a natural way

$$
\|f\|_{L_{\mu}^{p, \alpha}(\Gamma)}=\|f \mu\|_{L^{p, \alpha}(\Gamma)}, f \in L_{\mu}^{p, \alpha}(\Gamma) .
$$

The embedding $L^{p, \alpha_{1}}(\Gamma) \subset L^{p, \alpha_{2}}(\Gamma)$ is valid for $0 \leq \alpha_{1} \leq \alpha_{2} \leq 1$. Thus, $L^{p, \alpha}(\Gamma) \subset L_{1}(\Gamma), \forall \alpha \in[0,1]$, $\forall p \geq 1$. The case of $\Gamma \equiv[-\pi, \pi]$ will be denoted by $L^{p, \alpha}(-\pi, \pi) \equiv L^{p, \alpha}$.

By $S_{\Gamma}$ we denote the following singular integral operator

$$
\left(S_{\Gamma} f\right)(\tau)=\frac{1}{2 \pi i} \int_{\Gamma} \frac{f(\zeta) d \zeta}{\zeta-\tau}, \tau \in \Gamma .
$$

Unit circle centered at $z=0$ will be denoted by $\gamma$ with int $\gamma=\omega$. Define the Morrey-Hardy space $H_{+}^{p, \alpha}$ of functions $f(z)$ analytic inside $\omega$ with a norm $\|\cdot\|_{H_{+}^{p, \alpha}}$ :

$$
\|f\|_{H_{+}^{p, \alpha}}=\sup _{0<r<1}\left\|f\left(r e^{i t}\right)\right\|_{L^{p, \alpha}} .
$$

Denote by $\tilde{L}^{p, \alpha}$ the linear subspace of $L^{p, \alpha}$ consisting of functions whose shifts are continuous in $L^{p, \alpha}$, i.e. $\|f(\cdot+\delta)-f(\cdot)\|_{L^{p, \alpha}} \rightarrow 0$ as $\delta \rightarrow 0$. The closure of $\tilde{L}^{p, \alpha}$ in $L^{p, \alpha}$ will be denoted by $\mathcal{M}^{p, \alpha}$.

When studying non-homogeneous Riemann boundary value problem, we will essentially use the following lemma from [46].

Lemma 2.1. [46] Let $f(\cdot) \in L_{\infty} ; g(\cdot) \in \mathcal{M}^{p, \alpha} \wedge 1 \leq p<+\infty, 0<\alpha \leq 1$. Then the inclusion $f(\cdot) g(\cdot) \in \mathcal{M}^{p, \alpha}$ is valid.

Consider the following singular operator

$$
(S f)(\tau)=\frac{1}{2 \pi i} \int_{\gamma} \frac{f(\xi) d \xi}{\xi-\tau}, \tau \in \gamma .
$$

Using the results of $[13,14,16]$, it is easy to prove the following

Theorem 2.1. Singular operator $S$ acts boundedly in $\mathcal{M}^{p, \alpha}(\gamma)$ when $0<\alpha \leq 1$ and $1<p<+\infty$.

The following theorem can also be proved.

Theorem 2.2. Let $f \in \mathcal{M}^{p, \alpha}, 0<\alpha \leq 1,1<p<+\infty$. Then

where $(\mathcal{K} f)(z)$ is a Cauchy type integral

$$
\left\|(\mathcal{K} f)(r \xi)-f^{+}(\xi)\right\|_{L^{p, \alpha}} \rightarrow 0, r \rightarrow 1-0,
$$

$$
(\mathcal{K} f)(z)=\frac{1}{2 \pi i} \int_{\gamma} \frac{f(\xi) d \xi}{\xi-z}, z \notin \gamma .
$$


The similar assertion is also true for $f^{-}(\xi)$ as $r \rightarrow 1+0$, where $f^{-}(\cdot)$ are nontangential boundary values of $f(\cdot)$ outside $\omega$.

Consider the space $H_{+}^{p, \alpha}$. Denote by $L_{+}^{p, \alpha}$ the subspace of $L^{p, \alpha}$, generated by the restrictions of the functions from $H_{+}^{p, \alpha}$ to $\gamma$. It follows directly from the above results that the spaces $H_{+}^{p, \alpha}$ and $L_{+}^{p, \alpha}$ are isomorphic and $f^{+}(\tau)=(J f)(z)$, where $f \in H_{+}^{p, \alpha}, f^{+}$are the nontangential boundary values of $f$ on $\gamma$, and $J$ performs a corresponding isomorphism. Let $\mathcal{M}_{+}^{p, \alpha}=\mathcal{M}^{p, \alpha} \bigcap L_{+}^{p, \alpha}$. It is clear that $\mathcal{M}_{+}^{p, \alpha}$ is a subspace of $\mathcal{M}^{p, \alpha}$ with regard to the norm $\|\cdot\|_{L^{p, \alpha}}$. Let $\mathcal{M} H_{+}^{p, \alpha}=J^{-1}\left(\mathcal{M}_{+}^{p, \alpha}\right)$. This is a subspace of $H_{+}^{p, \alpha}$. Let $f \in H_{+}^{p, \alpha}$ and $f^{+}$be its boundary values. It is absolutely clear that the norm $\|f\|_{H_{+}^{p, \alpha}}$ can be also defined as $\|f\|_{H_{+}^{p, \alpha}}=\left\|f^{+}\right\|_{L^{p, \alpha}}$.

Similar to the classical case, we define the Morrey-Hardy class outside $\omega$. So let $D=C \backslash \omega$. We will say that the function $f$ analytic in $D^{-}$has finite order $k$ at infinity, if its Laurent series in a neighborhood of the point at infinity has the following form

$$
f(z)=\sum_{n=-\infty}^{k} a_{n} z^{n}, k<+\infty, a_{k} \neq 0 .
$$

Thus, when $k>0$, the function $f(z)$ has a pole of order $k$; when $k=0$, it is bounded; and when $k<0$, it has a zero of order $(-k)$. Let $f(z)=f_{0}(z)+f_{1}(z)$, where $f_{0}(z)$ is the main, and $f_{1}(z)$ is the regular part of expansion (1) for the function $f(z)$. Consequently, if $k \leq 0$, then $f_{0}(z) \equiv 0$. When $k>0, f_{0}(z)$ is a polynomial of degree $k$. We will say that the function $f(z)$ belongs to the class ${ }_{m} H_{-}^{p, \alpha}$, if $f$ has an order at infinity less than or equal to $m$, i.e. $k \leq m$ and $f_{1}\left(\frac{1}{z}\right) \in H_{+}^{p, \alpha}$.

Absolutely similar to the case of $\mathcal{M} H_{+}^{p, \alpha}$, we define the class ${ }_{m} \mathcal{M} H_{-}^{p, \alpha}$. In other words, ${ }_{m} \mathcal{M} H_{-}^{p, \alpha}$ is a subspace of functions from ${ }_{m} H_{-}^{p, \alpha}$, whose shifts on a unit circle are continuous with regard to the norm $\|\cdot\|_{L^{p, \alpha}(\gamma)} \cdot$

In the study of the basicity of the system of exponents, we will need the following result of [15].

Theorem 2.3. Systems $\left\{e^{i n t}\right\}_{n \in Z_{+}} ;\left\{e^{-i n t}\right\}_{n \in N}\left(\left\{z^{n}\right\}_{n \in Z_{+}} ;\left\{z^{-n}\right\}_{n \in N}\right)$ form bases for spaces $\mathcal{M}_{+}^{p, \alpha}$; ${ }_{-1} \mathcal{M}{ }_{-}^{p, \alpha}\left(\mathcal{M} H_{+}^{p, \alpha} ;{ }_{-1} \mathcal{M} H_{-}^{p, \alpha}\right)$, respectively.

We will also use the following concepts. Let $\Gamma \subset C$ be some bounded rectifiable curve, and $t=$ $t(\sigma), 0 \leq \sigma \leq l$, be its parametric representation with respect to the length of arc $\sigma$, where $l$ is the length of $\Gamma$. Let $d \mu(t)=d \sigma$, i.e. $\mu(\cdot)$ is a linear measure on $\Gamma$. Let

$$
\Gamma_{t}(r)=\{\tau \in \Gamma:|\tau-t|<r\}, \Gamma_{t(s)}(r)=\{\tau(\sigma) \in \Gamma:|\sigma-s|<r\} .
$$

It is absolutely clear that $\Gamma_{t(s)}(r) \subset \Gamma_{t}(r)$.

Definition 2.1. Curve $\Gamma$ is called a Carleson curve if $\exists c>0$ :

$$
\sup _{t \in \Gamma} \mu\left(\Gamma_{t}(r)\right) \leq c r, \forall r>0 .
$$

Curve $\Gamma$ is said to satisfy the chord-arc condition at the point $t_{0}=t\left(s_{0}\right) \in \Gamma$, if there exists a constant $m>0$, independent of $t$, such that $\left|s-s_{0}\right| \leq m\left|t(s)-t\left(s_{0}\right)\right|, \forall t(s) \in \Gamma$. $\Gamma$ satisfies the chord-arc condition uniformly on $\Gamma$, if $\exists m>0:|s-\sigma| \leq m|t(s)-t(\sigma)|, \forall t(s), t(\sigma) \in \Gamma$.

Let's state the following lemma from [16], which is interesting in itself.

Lemma 2.2. [16] Let $\Gamma$ be a bounded rectifiable curve. If the exponential function $\left|t-t_{0}\right|^{\gamma}, t_{0} \in \Gamma$, belongs to the space $L^{p, \alpha}(\Gamma), 1 \leq p<\infty, 0<\alpha<1$, then $\gamma \geq-\frac{\alpha}{p}$. If $\Gamma$ is a Carleson curve, then this condition is also sufficient.

We will essentially use the following theorem of N. Samko [16].

Theorem 2.4. Let the curve $\Gamma$ satisfy the chord-arc condition and the weight $\rho(\cdot)$ be defined as follows

$$
\rho(t)=\prod_{k=1}^{m}\left|t-t_{k}\right|^{\alpha_{k}} ;\left\{t_{k}\right\}_{1}^{m} \subset \Gamma, t_{i} \neq t_{j}, i \neq j .
$$


Singular operator $S_{\Gamma}$ is bounded in the weighted space $L_{\rho}^{p, \alpha}(\Gamma), 1<p<+\infty, 0 \leq \alpha<1$, if the following inequalities are valid

$$
-\frac{\alpha}{p}<\alpha_{k}<-\frac{\alpha}{p}+1, k=\overline{1, m} .
$$

Moreover, if $\Gamma$ is smooth in some neighborhoods of the points $t_{k}, k=\overline{1, m}$, then the validity of the inequalities (5) is necessary for the boundedness of $S_{\Gamma}$ in $L_{\rho}^{p, \alpha}(\Gamma)$.

In what follows, as $\Gamma$ we will consider a unit circle $\gamma=\partial \omega$. Consider the weighted space $L_{\rho}^{p, \alpha}(\gamma)=: L_{\rho}^{p, \alpha}$ with the weight $\rho(\cdot)$. Let the weight $\rho(\cdot)$ satisfy the condition (5). Then, by Theorem 2.4 , the operator $S$ is bounded in $L_{\rho}^{p, \alpha}$, i.e. $\exists c>0$ :

$$
\|S f\|_{L_{\rho}^{p, \alpha}} \leq C\|f\|_{L_{\rho}^{p, \alpha}}, \forall f \in L_{\rho}^{p, \alpha} .
$$

\section{Basicity of the Perturbed System of Exponents in Morrey-Lebesgue Space}

Consider the following system of exponents

$$
\left\{e^{i(n+\beta s i g n n) t}\right\}_{n \in Z} .
$$

Let us represent the following type system:

$$
\left\{A(t) e^{i n t} ; B(t) e^{-i k t}\right\}_{n \in Z_{+}, k \in N},
$$

where

$$
A(t)=e^{i \beta t} ; B(t)=e^{-i \beta t},
$$

on an interval $[-\pi, \pi]$, where $Z_{+}=\{0\} \cup N$.

Let

$$
G\left(e^{i t}\right)=\frac{A(t)}{B(t)}, t \in[-\pi, \pi] .
$$

Take $\forall f \in \mathcal{M}^{p, \alpha}$ and consider the following Riemann boundary value problem in classes $\mathcal{M} H_{+}^{p, \alpha} \times$ ${ }_{-1} \mathcal{M} H_{-}^{p, \alpha}$ :

$$
F^{+}(\tau)-G(\tau) F^{-}(\tau)=A^{-1}(\arg \tau) f(\arg \tau), \tau \in \gamma .
$$

Assume that the following inequalities are fulfilled

$$
-\frac{1-\alpha}{q} \leq \beta<\frac{1-\alpha}{p}
$$

We will apply Theorem 10 [46] to the solution of the problem (7). From Lemma 2.1 follows that the function $A^{-1}(\cdot) f(\cdot)$ belongs to the space $\bar{L}^{p, \alpha}$. Therefore, if the inequality (8) are fulfilled, as it follows from Theorem 10 [46] the problem (7) is uniquely solvable in class $\mathcal{M} H_{+}^{p, \alpha} \times_{-1} \mathcal{M} H_{-}^{p, \alpha}$, and this solution can be represented as an integral

$$
F(z)=\frac{Z(z)}{2 \pi} \int_{-\pi}^{\pi} \frac{f(t)}{A(t) Z^{+}\left(e^{i t}\right)} \frac{d t}{1-z e^{-i t}} .
$$

Absolutely obvious that the following inclusion is true

$$
F^{+}(\cdot) \in \mathcal{M}_{+}^{p, \alpha} ; F^{-}(\cdot) \in{ }_{-1} \mathcal{M}_{-}^{p, \alpha} \text {. }
$$

By Theorem 2.3, systems $\left\{e^{i n t}\right\}_{n \in Z_{+}},\left\{e^{-i n t}\right\}_{n \in N}$ form a basis for spaces $\mathcal{M}_{+}^{p, \alpha}$ and ${ }_{-1} \mathcal{M}_{-}^{p, \alpha}$, respectively. Let us expand the functions $F^{+}(\cdot)$ and $F^{-}(\cdot)$ on these systems. We have 


$$
F^{+}\left(e^{i t}\right)=\sum_{n=0}^{\infty} a_{n} e^{i n t} ; F^{-}\left(e^{i t}\right)=\sum_{n=1}^{\infty} b_{n} e^{i n t} .
$$

Taking into account these decompositions in $(7)$ we obtain, that the function $f(\cdot)$ has an expansion on the system (6) in $\mathcal{M}^{p, \alpha}$ :

$$
f(t)=A(t) \sum_{n=0}^{\infty} a_{n}^{+} e^{i n t}+B(t) \sum_{n=1}^{\infty} a_{n}^{-} e^{-i n t},
$$

where $a_{n}^{+}=a_{n}, a_{n}^{-}=-b_{n}$. Let us find an expression for the coefficients $\left\{a_{n}^{ \pm}\right\}$. Let

$$
Z(z)=\sum_{n=0}^{\infty} c_{n}^{+} z^{n}, \quad|z|<1,
$$

be a Taylor expansion of the function $Z(\cdot)$ in the neighborhood of the point $z=0$. We have

$$
\frac{1}{2 \pi} \int_{-\pi}^{\pi} \frac{f(t)}{A(t) Z^{+}\left(e^{i t}\right)} \frac{d t}{1-z e^{-i t}}=\sum_{n=0}^{\infty} d_{n}^{+} z^{n}
$$

where

$$
d_{n}^{+}=\frac{1}{2 \pi} \int_{-\pi}^{\pi} \frac{e^{-i n t}}{A(t) Z^{+}\left(e^{i t}\right)} f(t) d t, n \in Z_{+} .
$$

As a result, for $F(\cdot)$ we have the decomposition

$$
F(z)=\sum_{n=0}^{\infty} A_{n}^{+} z^{n},|z|<1,
$$

where $A_{n}^{+}=\sum_{k=0}^{n} c_{n-k}^{+} d_{k}^{+}$. Taking into account the expression for $d_{n}^{+}$we have

$$
A_{n}^{+}=\int_{-\pi}^{\pi} \overline{v_{n}^{+}(t)} f(t) d t
$$

where

$$
v_{n}^{+}(t)=\frac{1}{2 \pi} \sum_{k=0}^{n} \bar{c}_{n-k}^{+} \frac{e^{+i k t}}{\overline{A(t) Z^{+}\left(e^{i t}\right)}}, n \in Z_{+} .
$$

Similarly, let us expand the function $F(\cdot)$ in a Taylor series in the neighborhood of $z=\infty$. Again, using the expression for $F(\cdot)$, we have

$$
Z(z)=\sum_{n=0}^{\infty} c_{n}^{-} z^{-n},|z|>1 .
$$

Also we have

$$
\frac{1}{2 \pi} \int_{-\pi}^{\pi} \frac{f(t)}{A(t) Z^{+}\left(e^{i t}\right)} \frac{d t}{1-z e^{-i t}}=\sum_{n=1}^{\infty} d_{n}^{-} z^{-n},
$$

where

$$
d_{n}^{-}=-\frac{1}{2 \pi} \int_{-\pi}^{\pi} \frac{e^{i n t}}{A(t) Z^{+}\left(e^{i t}\right)} f(t) d t, n \in N .
$$

So, $F(\cdot)$ has an expansion 


$$
F(z)=\sum_{n=1}^{\infty} A_{n}^{-} z^{-n},|z|>1
$$

where

$$
A_{n}^{-}=\sum_{k=1}^{n} c_{n-k}^{-} d_{k}^{-} .
$$

Similar to the previous case for $A_{n}^{-}$we have $A_{n}^{-}=\int_{-\pi}^{\pi} \overline{v_{n}^{-}(t)} f(t) d t$, where

$$
v_{n}^{-}(t)=-\frac{1}{2 \pi} \sum_{k=1}^{n} \bar{c}_{n-k}^{-} \frac{e^{-i k t}}{\overline{A(t) Z^{+}\left(e^{i t}\right)}}, n \in N .
$$

On the other hand, it is easy to see that

$$
\begin{gathered}
a_{n}^{+}=\frac{1}{2 \pi} \int_{-\pi}^{\pi} F^{+}\left(e^{i t}\right) e^{-i n t} d t \\
A_{n}^{+}=\frac{1}{2 \pi r^{n}} \int_{-\pi}^{\pi} F\left(r e^{i t}\right) e^{-i n t} d t, 0<r<1, \forall n \in Z_{+} .
\end{gathered}
$$

are true. Then from Theorem 2.2 it directly follows that $A_{n}^{+}=a_{n}^{+}, \forall n \in Z_{+}$. Similarly we obtain $A_{n}^{-}=a_{n}^{-}$, $\forall n \in N$.

And now, as $f(t)$ we take $f(t) \equiv A(t) e^{i n_{0} t}$, where $n_{0} \in Z_{+}$is an arbitrary fixed number. In this case the solution of the problem (7) is the following function

$$
\Phi^{+}(z)= \begin{cases}z^{n_{0}}, & |z|<1 \\ 0, & |z|>1\end{cases}
$$

As it follows from Theorem 10 [46], the problem (7) is uniquely solvable in classes $\mathcal{M} H_{+}^{p, \alpha} \times{ }_{1} \mathcal{M} H_{-}^{p, \alpha}$. Therefore, comparing functions $F(\cdot)$ and $\Phi^{+}(\cdot)$, we have

$$
A_{n}^{+}=\left\{\begin{array}{ll}
1, & n=n_{0}, \\
0, & n \neq n_{0},
\end{array} A_{n}^{-}=0, \forall n \in N\right.
$$

i.e., we have

$$
\begin{gathered}
\int_{-\pi}^{\pi} A(t) e^{i n_{0} t} \bar{v}_{n}(t) d t=\delta_{n n_{0}}, \forall n, n_{0} \in Z_{+}, \\
\int_{-\pi}^{\pi} A(t) e^{i n_{0} t} \bar{v}_{n}^{-}(t) d t=0, \forall n_{0} \in Z_{+}, \forall n \in N .
\end{gathered}
$$

Similarly, taking $f(t)=B(t) e^{-i n_{0} t}$, we obtain that in this case the solution of the problem (7) is also the following function

$$
\Phi^{-}(z)= \begin{cases}0, & |z|<1 \\ z^{-n_{0}}, & |z|>1\end{cases}
$$

Again, from the similar considerations we obtain

$$
\begin{gathered}
\int_{-\pi}^{\pi} B(t) e^{-i n_{0} t} \bar{v}_{n}^{+}(t) d t=0, \forall n_{0} \in N, \forall n \in Z_{+} ; \\
\int_{-\pi}^{\pi} B(t) e^{-i n_{0} t} \bar{v}_{n}^{-}(t) d t=\delta_{n n_{0}}, \forall n, n_{0} \in N .
\end{gathered}
$$

From these relations it follows that if the inclusion $\left\{v_{n}^{+} ; v_{n}^{-}\right\}_{n \in Z_{+}, k \in N} \subset\left(\mathcal{M}^{p, \alpha}\right)^{*}$ is true, then the system (6) is minimal in $\mathcal{M}^{p, \alpha}$. It suffices to show that 


$$
v_{n}^{+} ; v_{k}^{-} \in L^{q, \alpha}, \forall n \in Z_{+}, \forall k \in N
$$

We have

$$
\left|Z^{+}\left(e^{i t}\right)\right|^{-1} \sim|t-\pi|^{\beta}, t \in[-\pi, \pi] .
$$

Let the inequality

$$
\beta \geq \frac{\alpha-1}{q},
$$

be fulfilled. Then by Lemma 2.2 it follows that $\left|Z^{+}\left(e^{i t}\right)\right|^{-1}$ belongs to $L^{q, \alpha}$. Hence, from the expressions for $v_{n}^{ \pm}$follows the inclusion $\left\{v_{n}^{ \pm}\right\} \subset L^{q, \alpha}$. As a result we have that the system (6) is minimal in $\mathcal{M}^{p, \alpha}$. It is clear that the function $f(\cdot)$ has a unique expansion of the form (9) on this system. Thus, the following theorem is true.

Theorem 3.1. Let the inequality

$$
-\frac{1}{q}+\frac{\alpha}{q} \leq \beta<\frac{1}{p}-\frac{\alpha}{p}
$$

be fulfilled, then the system (6) forms a basis for $\mathcal{M}^{p, \alpha}, 1<p<+\infty, 0<\alpha<1$.

\section{References}

1. A. L. Mazuccato, "Decomposition of besov-morrey spaces. in harmonic analysis at mount holyoke," $A M S$ series in Contemporary Mathematics, vol. 320, pp. 279-294, 2003.

2. Y. Chen, "Regularity of the solution to the dirichlet problem in morrey space," J. Partial Diff. Equations, vol. 15, pp. 37-46, 2002.

3. A. Akbulut and O. Kuzu, "Marcinkiewicz integrals with rough kernel associated with schrodinger operator on vanishing generalized morrey spaces," Azerb. J. of Math., vol. 4, no. 1, pp. 40-54, 2014.

4. P. G. Lemarie-Rieusset, "Some remarks on the navier-stokes equations in $r^{3}$," J. Math. Phys., vol. 39, no. 8, pp. 4108-4118, 1988.

5. Y. Giga and T. Miyakawa, "Navier-stokes flow in $r^{3}$ with measures as initial vorticity and morrey spaces," Comm. In Partial Differential Equations, vol. 14, no. 5, pp. 577-618, 1989.

6. J. Duoandikoetxea, Weight for maximal functions and singular integrals. NCTH Summer School on, Harmonic Analysis in Taiwan, 2005.

7. J. Peetre, "On the theory of $l^{p, \lambda}$ spaces," J. Funct. Anal., vol. 4, pp. 71-87, 1964.

8. C. T. Zorko, "Morrey space," Proc. of the Amer. Math. Society, vol. 98, no. 4, pp. 586-592, 1986.

9. R. C. Mustafayev, "On boundedness of sublinear operators in weighted morrey spaces," Azerb. J. of Math., vol. 2, no. 1, pp. 66-79, 2012.

10. V. S. Guliyev, "Local generalized morrey spaces and singular integrals with rough kernel," Azerb. J. of Math., vol. 3, no. 2, pp. 79-94, 2013.

11. V. S. Guliyev and M. N. Omarova, "Multilinear singular and fractional integral operators on generalized weighted morrey spaces," Azerb. J. of Math., vol. 5, no. 1, pp. 104-132, 2015.

12. N. X. Ky, "On approximation by trigonometric polynomials in $l_{u^{-}}^{p}$ spaces," Studia Sci. Math. Hungar, vol. 28, pp. 183-188, 1993.

13. V. Kokilashvili and A. Meskhi, "Boundedness of maximal and singular operators in morrey spaces with variable exponent," Govern. College Univ., Lahore, vol. 72, pp. 1-11, 2008.

14. D. M. Israfilov and N. P. Tozman, "Approxiamtion by polynomials in morrey-smirnov classes," East J. Approx., vol. 14, no. 3, pp. 255-269, 2008.

15. B. T. Bilalov and A. A. Quliyeva, "On basicity of exponential systems in morrey type spaces," Int. J. of Math., vol. 25, no. 6, pp. 1-10, 2014.

16. N. Samko, "Weight hardy and singular operators in morrey spaces," J. of Math. Anal. and Appl., vol. 35, no. 1, pp. 183-188, 2009.

17. D. M. Israfilov and N. P. Tozman, "Approximation in morrey-smirnov classes," Azerb. J. of Math., vol. 1, no. 1, pp. 99-113, 2011.

18. I. I. Sharapudinov, "On direct and inverse theorems of approximation theory in variable lebesgue and sobolev spaces," Azerb. J. of Math., vol. 4, no. 1, pp. 55-72, 2014. 
19. B. Bilalov and F. Guliyeva, "On the basis properties of the systems in the intuitionistic fuzzy metric space," Azerb. J. of Math., vol. 4, no. 1, pp. 136-149, 2014.

20. S. M. Ponomarev, "On an eigen value problem," Doklady Akademii Nauk SSSR, vol. 249, no. 5, pp. 1068-1070, 1979.

21. E. I. Moiseev, "On some boundary value problems for mixed type equations," Diff Uravn., vol. 28, no. 1, pp. 123-132, 1992.

22. —_ "On solution of frankle's problem in special domain," Diff Uravn., vol. 28, no. 4, pp. 682-692, 1992.

23. - "On existence and uniqueness of solution a classical problem," Doklady Rossiyskaya Akademiya Nauk, vol. 336, no. 4, pp. 448-450, 1994.

24. R. Paley and N. Wiener, "Fourier transforms in the complex domain," Amer. Math. Soc. Colloq. Publ. (Amer. Math. Soc., Providence, RI, vol. 19, 1934.

25. N. Levinson, "Gap and density theorems," Amer. Math. Soc. Colloq. Publ. (Amer. Math. Soc., Providence, RI, vol. 29, 1940 .

26. A. M. Sedletskii, "Biorthogonal expansions in series of exponents on intervals of real axis," Usp. Mat. Nauk, vol. 37:5, no. 227, pp. 51-95, 1982.

27. E. I. Moiseev, "On basicity of the system of sines and cosines," DAN SSSR, vol. 275, no. 4, pp. 794-798, 1984.

28. — - "On basicity of a system of sines," Diff. Uravn., vol. 23, no. 1, pp. 177-179, 1987.

29. B. T. Bilalov, "Basicity of some systems of exponents, cosines and sines," Diff. Uravn., vol. 26, no. 1, pp. 10-16, 1990.

30. — - "Basis properties of some systems of exponents, cosines and sines," Sib. Math. Journal, vol. 45, no. 2, pp. 264-273, 2004.

31. — "Property of basicity in lp of the system of exponents," Sib. Math. Journal, vol. 47, no. 1, 2006.

32. - "A system of exponential functions with shift and the kostyuchenko problem," Sib. Math. Journal, vol. 50, no. 2, pp. 279-288, 2009.

33. —_ "On solution of the kostyuchenko problem," Sib. Math. Journal, vol. 53, no. 3, pp. 509-526, 2012.

34. E. I. Moiseev, "On basicity of systems of cosines and sines in weight space," Diff. Uravn., vol. 34, no. 1, pp. 40-44, 1998.

35. - " "The basicity in the weight space of a system of eigen functions of a differential operator," Diff. Uravn., vol. 35, no. 2, pp. 200-205, 1999.

36. — - "On differential properties of expansions on the system of sines and cosines," Diff. Uravn., vol. 32, no. 1, pp. 117-126, 1996.

37. A. M. Sedletskii, "Approximate properties of the system of exponents in sobolev spaces," Vestnik Moskovskogo Universiteta, vol. 6, pp. 3-8, 1999.

38. Z. G. Huseynov and A. M. Shykhammedov, "On bases of sines and cosines in sobolev spaces," Applied Mathematics Letters, vol. 25, pp. 275-278, 2012.

39. D. L. Russell, "On exponential bases for the sobolev spaces over an interval," Journal of Math. Analysis and Applications, vol. 87, pp. 528-550, 1982.

40. X. He and H. Volkmer, "Riesz bases of solutions of sturm-lioville equations," J.Fourier Anal. Appl., vol. 7, no. 3, pp. 297-307, 2001.

41. B. T. Bilalov, "On basicity of the system of exponents, cosines and sines in lp," Dokl. RAN, vol. 365, no. 1, pp. 7-8, 1999.

42. —_ , "On basicity of some systems of exponents, cosines and sines in lp," Dokl. RAN, vol. 379, no. 2, pp. 7-9, 2001.

43. - "Bases of exponents, cosines and sines which are eigenfunctions of differential operators," Dif. Uravn., vol. 39, no. 5, pp. 1-5, 2003.

44. T. I. Najafov and N. P. Nasibova, "On the noetherness of the riemann problem in generalized weighted hardy classes," Azerb. J. of Math., vol. 5, no. 2, pp. 109-124, 2015.

45. A. V. Bitsadze, "On a system of functions," $U M N$, vol. 5, no. 4:38, pp. 150-151, 1950.

46. B. T. Bilalov, T. B. Gasymov, and A. A. Guliyeva, "On solvability of riemann boundary value problem in morrey-hardy classes," Turk. J. Math., vol. 40, pp. 1085-1101, 2016. 